\title{
Optimal Slot Allocation Method for ITE Systems
}

\author{
Bin Zeng \\ Dept. management engineering, Naval university of \\ engineering, Wuhan, China \\ E-mail: zbtrueice@163.com
}

\author{
Rui Wang \\ Library, Dept. training, Naval university of engineering \\ Wuhan, China \\ E-mail: kingwis@163.com
}

\begin{abstract}
To achieve better performance and bandwidth in the mobile communication, some vendors have already introduced LTE service. The following issues concerning LTE such as performance estimation, throughput analysis, quality of service, voice/data integration are of great interest for deep research. Therefore, a mathematical model for finding the best policy of slot allocation is proposed. Since channels (or slots) are shared by data and voice users and can be dynamically allocated to LTE users, there must be a lot of policies can be applied to do this. The system revenue under some assumption will be maximized in this scheduling method. The mathematical formulation and solution approach for this problem is also discussed. And some computational results are given finally.
\end{abstract}

Keywords-Long term evolution; Optimal algorithm; Slot allocation; Markov Chain Model

\section{INTRODUCTION}

In order to satisfy the increasing user requirements and to preserve competitiveness, in developing of $4 \mathrm{G}$, the $3 \mathrm{GPP}$ has specified Long Term Evolution (LTE) that accommodates data connections with high bandwidth efficiency.

While basing on 4G, LTE introduces new components and new protocols to support packet switching, but it still use original packet channels and some techniques [1]. An important property of LTE is that the LTE and packet switch services shared the same radio resources. When the voice channels are unused by packet switching service, they will be allocated dynamically to the LTE according to the actual needs for packet transfers.

This mechanism brings up some issues for research. Since channels (or slots) are shared by data and voice users and can be dynamically allocated to LTE users, there must be a lot of policies can be applied to do this [2]. For example, we can reserve some channels for voice users to prevent blocking. We also can reserve channels for LTE users to offer better quality of service. When channels are all in use, we can determine the release action: immediate release or delayed release. Different policy causes different system performance, and different system revenue.

In the vender's point of view, revenue maximization or cost minimization is the top concern. So in this paper, our goal is to find a best policy of slot allocation that can maximize system revenue.

In this paper, we want to develop the model that can describe the slot allocation system. And we will show how the problem can be solved to find the best policy.

We model the slot allocation problem as a Markov process. This research is extended from [3]. Reference [3] is a LTE performance estimation. It assumes that voice and LTE user arrivals can be characterized by a Poisson process, and the service times of users are exponentially distributed. So in this paper we use the same assumption. But in [3] the model only consider data users that only need one slot service. It uses simulation to deal with multiple slot service. And it computes the average blocking probability, throughput and average queuing time.

In this paper, we consider three kinds of users: voice users, single slot data users and two-slot data users. Because LTE uses and voice users shared the same channel and there are eight slots in one channel, we use eight slots to model the problem.

\section{PROBLEM DESCRIPTION}

There are two ways to describe our problem. This paper will use both methods to solve the problem. Our model is a LTE revenue optimization model. Due to TDMA technique, there are eight time slots in one channel. Consequently, there are up to eight single-slot users that can transmit data at the same time. However, this paper also considers some users that need two-slot transmission. So total user number in one channel may be less than eight [4]. We consider three classes of traffic, voice user, single-slot data user, and two-slot data user. Although other kind of users is also possible, we only model these users in this paper. We have eight decisions in this paper shown as Table I.

\section{TABLE I. DECISIONS AND ACTION MATRIX}

\begin{tabular}{|l|l|}
\hline $\begin{array}{l}\text { Decision } \\
k\end{array}$ & Action \\
\hline 1 & Grant all users \\
2 & Grant voice and single-slot data users \\
3 & Grant one-slot data and two-slot data users \\
4 & Grant voice and two-slot data users \\
5 & Only grant voice users \\
6 & Only grant single-slot data users \\
7 & Only grant two-slot data users \\
8 & Do not grant any users \\
\hline
\end{tabular}

We define the system state as $(a, b, c)$ : a means the number of voice users, $b$ means the number of single-slot data users, and c means the number of two-slot data users. For example, $(2,3,1)$ means there are 2 voice users, 3 singleslot data users, and 1 two-slot data user in the system. Since total numbers of slots are 8,1 slot is free for use $(2+3+1 * 2=7$, $8-7=1)$

We have three assumptions in our model in this chapter:

1. Both voice and data messages arrivals follow a Poisson process and call holding time and data message length are 
exponentially distributed with different parameters. Singleslot data users and two-slot data users are independent.

2. For each voice users, only one slot is dedicated. For data users, one or two slots are dedicated [5].

3. A slot is called a channel. There are in total 8 channels in our model.

Fig. 1 is the 3-dimensional Markov chain model of our problem, and Fig. 2 is the details of a triangle in Fig. 1.

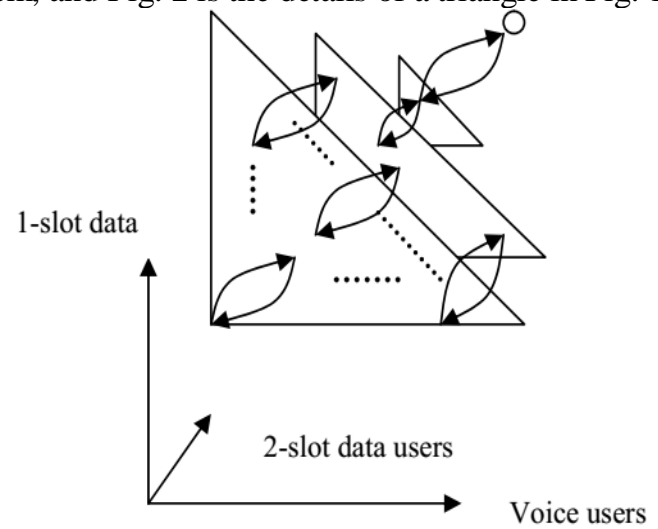

Figure 1. 3-dimensional Markov Chain Model

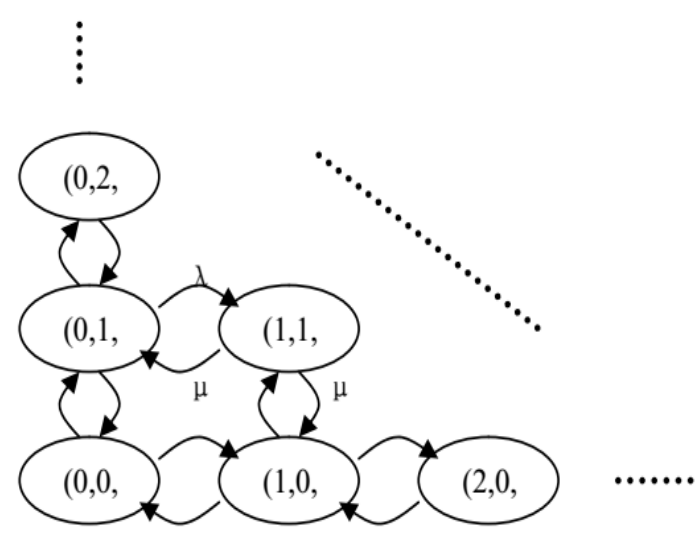

Figure 2. Details of a Triangle in Figure 3-1

We considered the problem as below. Given: Voice users arrival rate, Voice users service rate, Data users need one-slot arrival rate, Data users need one-slot service rate, Data users need two-slot arrival rate, Data users need two-slot service rate, and System revenue according to the decision and action. We should determine the best slot allocation policy. The objective is to maximize the system revenue subject to the slot capacity constraint.

The notations we use in this paper are shown as below. $\lambda 1$ denotes the arrival rate of voice users, $\lambda 2$ denotes the arrival rate of single-slot users, $\lambda 3$ denotes the arrival rate of two-slot users, $\mu 1$ denotes The service rate of voice users, $\mu 2$ denotes the service rate of single-slot data users, $\mu 3$ denotes The service rate of two-slot data users, R1 denotes the system revenue of servicing one voice user, R2 denotes the system revenue of servicing one single-slot user, R3 denotes the system revenue of servicing one two-slot user, aijk denotes The appropriate transition rate from state $i$ to state $j$ given decision $\mathrm{k}$.

\section{MATHEMATIC FORMULATION}

The first policy of our model is that does not preserve any slot in advance and use immediate release action. As long as the system is not full and can handle the traffic, coming users can use the resource. If voice users come and find there are no free slots, data users must stop their transmission and wait until some slots are free. In this policy, there are total 95 states. Other policies will decrease numbers of states. So in our model there are at most 95 states. Table II is the sequence of the state of our model. As mentioned in Section II, we will use continuous-time Markov decision process to solve our problem

Because it is a completely erotic processes in our model, so we use the simple method to find the best policy.

We use the equations

$$
g=q_{i}+\sum_{j=1}^{95} a_{i j} v_{j}
$$

Where

$$
q_{i}=r_{i i}+\sum_{j \neq i} a_{i j} r_{i j}
$$

The associated parameter aij and rij are given (the first policy). The parameter rii equals to zero in our model because the system earns nothing during all the time that it occupies state $\mathrm{i}$.

Form (2) we can compute the values of qi:

$\mathrm{q} 1=0$

$\mathrm{q} 2=\mu 3 \mathrm{R} 3$

$\mathrm{q} 3=\mu 3 \mathrm{R} 3$

:

$\mathrm{q} 32=\mu 1 \mathrm{R} 1+\mu 2 \mathrm{R} 2+\mu 3 \mathrm{R} 3$

$\mathrm{q} 95=\mu 1 \mathrm{R} 1$

From (1) we can write the first equation of the formulation:

$\mathrm{g}=0+(-\lambda 1-\lambda 2-\lambda 3) \mathrm{v} 1+\lambda 3 \mathrm{v} 2+\lambda 2 \mathrm{v} 6+\lambda 1 \mathrm{v} 26$

$\mathrm{g}=\mu 3 \mathrm{R} 3+\mu 3 \mathrm{v} 1+(-\lambda 1-\lambda 2-\lambda 3-\mu 3) \mathrm{v} 2+\lambda 3 \mathrm{v} 3+\lambda 2 \mathrm{v} 7$ $+\lambda 1 \mathrm{v} 27$

$\mathrm{g}=\mu 1 \mathrm{R} 1+\mu 1 \mathrm{v} 93$

Let $\mathrm{v} 95=0$. And we can solve $\mathrm{g}, \mathrm{v} 1, \mathrm{v} 2, \ldots, \mathrm{v} 94$. Then we go to the policy improvement step. For each state i, find the alternative $\mathrm{k}$ that maximizes

$$
q_{i}^{k}+\sum_{j=1}^{N} a_{i j}^{k} v_{j}
$$

using the relative values vi of the previous policy.

When we find the policy, we compare if it is the same policy. If it is not, we go back to the value determination step. Once the new policy and the old policy are the same, it's the optimal policy. 
TABLE II. THE SEQUENCE OF THE STATES

\begin{tabular}{|c|c|c|c|c|c|c|c|}
\hline 1 & $(0,0,0)$ & 25 & $(0,8,0)$ & 49 & $(2,0,3)$ & 73 & $(3,5,0)$ \\
\hline 2 & $(0,0,1)$ & 26 & $(1,0,0)$ & 50 & $(2,1,0)$ & 74 & $(4,0,0)$ \\
\hline 3 & $(0,0,2)$ & 27 & $(1,0,1)$ & 51 & $(2,1,1)$ & 75 & $(4,0,1)$ \\
\hline 4 & $(0,0,3)$ & 28 & $(1,0,2)$ & 52 & $(2,1,2)$ & 76 & $(4,0,2)$ \\
\hline 5 & $(0,0,4)$ & 29 & $(1,0,3)$ & 53 & $(2,2,0)$ & 77 & $(4,1,0)$ \\
\hline 6 & $(0,1,0)$ & 30 & $(1,1,0)$ & 54 & $(2,2,1)$ & 78 & $(4,1,1)$ \\
\hline 7 & $(0,1,1)$ & 31 & $(1,1,1)$ & 55 & $(2,2,2)$ & 79 & $(4,2,0)$ \\
\hline 8 & $(0,1,2)$ & 32 & $(1,1,2)$ & 56 & $(2,3,0)$ & 80 & $(4,2,1)$ \\
\hline 9 & $(0,1,3)$ & 33 & $(1,1,3)$ & 57 & $(2,3,1)$ & 81 & $(4,3,0)$ \\
\hline 10 & $(0,2,0)$ & 34 & $(1,2,0)$ & 58 & $(2,4,0)$ & 82 & $(4,4,0)$ \\
\hline 11 & $(0,2,1)$ & 35 & $(1,2,1)$ & 59 & $(2,4,1)$ & 83 & $(5,0,0)$ \\
\hline 12 & $(0,2,2)$ & 36 & $(1,2,2)$ & 60 & $(2,5,0)$ & 84 & $(5,0,1)$ \\
\hline 13 & $(0,2,3)$ & 37 & $(1,3,0)$ & 61 & $(2,6,0)$ & 85 & $(5,1,0)$ \\
\hline 14 & $(0,3,0)$ & 38 & $(1,3,1)$ & 62 & $(3,0,0)$ & 86 & $(5,1,1)$ \\
\hline 15 & $(0,3,1)$ & 39 & $(1,3,2)$ & 63 & $(3,0,1)$ & 87 & $(5,2,0)$ \\
\hline 16 & $(0,3,2)$ & 40 & $(1,4,0)$ & 64 & $(3,0,2)$ & 88 & $(5,3,0)$ \\
\hline 17 & $(0,4,0)$ & 41 & $(1,4,1)$ & 65 & $(3,1,0)$ & 89 & $(6,0,0)$ \\
\hline 18 & $(0,4,1)$ & 42 & $(1,5,0)$ & 66 & $(3,1,1)$ & 90 & $(6,0,1)$ \\
\hline 19 & $(0,4,2)$ & 43 & $(1,5,1)$ & 67 & $(3,1,2)$ & 91 & $(6,1,0)$ \\
\hline 20 & $(0,5,0)$ & 44 & $(1,6,0)$ & 68 & $(3,2,0)$ & 92 & $(6,2,0)$ \\
\hline 21 & $(0,5,1)$ & 45 & $(1,7,0)$ & 69 & $(3,2,1)$ & 93 & $(7,0,0)$ \\
\hline 22 & $(0,6,0)$ & 46 & $(2,0,0)$ & 70 & $(3,3,0)$ & 94 & $(7,1,0)$ \\
\hline 23 & $(0,6,1)$ & 47 & $(2,0,1)$ & 71 & $(3,3,1)$ & 95 & $(8,0,0)$ \\
\hline 24 & $(0,7,0)$ & 48 & $(2,0,2)$ & 72 & $(3,4,0)$ & & \\
\cline { 1 - 3 }
\end{tabular}

\section{SOLUTION PROCEDURE}

As mentioned in Section III, we will solve our problem using continuous Markov decision process. The transition rate is according to the Poisson distribution process. We will have only one revenue matrix because different policies have no influence on the revenue matrix.

To apply the policy iteration method, we must solve simultaneous equations [6]. To solve these equations, we use the Gauss elimination method to get the solution of each variable. We use backward substitution and partial pivot method to get the solution. Once we get the solution, we can apply the policy iteration method to find the best policy.

Define aij as the transition rates of a process from state $\mathrm{i}$ to state $\mathrm{j}$, for $\mathrm{i} \neq \mathrm{j}$. The quantity is defined as follows: In a short time interval $\mathrm{dt}$, a process that is now in state $\mathrm{i}$ will make a transition to state $\mathrm{j}$ with probability aij $\mathrm{dt}$ (ifj). We describe the continuous-time Markov Process by a transitionrate matrix.

Suppose that the system earns a reward at the rate of rij dollars per unit time during all the time that it occupies state i. Suppose further that when the system makes a transition from state $i$ to state $j(i \neq j)$ it receives a reward of rij.

Let vi(t) be the expected total reward that the system will earn in a time $t$ if it starts in state $i$. then we can relate the total expected reward in a time $t+d t$, vi( $t+d t)$, to vi(t) by

$$
v_{i}(t+d t)=\left(1-\sum_{j \neq i} a_{i j} d t\right)\left[r_{i i} d t+v_{i}(t)\right]+\sum_{j \neq i} a_{i j} d t\left[r_{i j}+v_{j}(t)\right]
$$

We can start to evaluate:

- Step 1: Value determination step:

We define a quantity qi as the "earning rate" of the system where

$$
q_{i}=r_{i i}+\sum_{j \neq i} a_{i j} r_{i j}
$$

For a given policy the total expected reward of system in time $t$ is governed by (6) with use of the definition of earning rate (5) to (4)

$$
\frac{d}{d t} v_{i}(t)=q_{i}+\sum_{j=1}^{N} a_{i j} v_{j}(t) \mathrm{i}=1,2, \ldots, \mathrm{N}
$$

Since we were concerned only with processes whose termination is remote, we may use the asymptotic expression for $\operatorname{vi}(\mathrm{t})$

$$
v i(t)=\operatorname{tgi}+v i, \text { for large } t
$$

Thus we have

$$
g_{i}=q_{i}+\sum_{j=1}^{N} a_{i j}\left(t_{j}+v_{j}\right), \mathrm{i}=1,2, \ldots, \mathrm{N}
$$

or

$$
g_{i}=q_{i}+t \sum_{j=1}^{N} a_{i j} g_{j}+\sum_{j=1}^{N} a_{i j} v_{j}, \mathrm{i}=1,2, \ldots, \mathrm{N}
$$

- Step 2: Policy iteration step:

For each state $\mathrm{i}$, determine the alternative $\mathrm{k}$ that

maximizes $\sum_{j=1}^{N} a_{i j}^{k} g_{j}$ using the gains gj of the previous policy, and make it the new decision in the ith state.

If $\sum_{j=1}^{N} a_{i j}^{k} g_{j}$ is the same for all alternatives, or if several alternatives are equally good according to this test, the decision must be made on the basis of relative values rather than gains. Therefore, if the gain test fails, break the tie by determining the alternative $\mathrm{k}$ that maximize ${ }^{q_{i}^{k}+\sum_{j=1}^{N} a_{i j}^{k} v_{j}}$ using the relative values of the previous policy, and by making it the new decision in the ith state. If the new policy is the same as the previous policy, then stop; otherwise, repeat the two steps.

\section{COMPUTATIONAL RESULTS}

We will use an example to demonstrate our problem and show the computational results of our model.

Given parameters are shown as below.

TABLE III. GIVEN PARAMETERS OF EXAMPLE

\begin{tabular}{|l|l|l|}
\hline \multicolumn{1}{|c|}{$\lambda_{1}=9$} & $\mu_{1}=8$ & $R_{1}=10$ \\
\hline$\lambda_{2}=3$ & $\mu_{2}=4$ & $R_{2}=2$ \\
\hline$\lambda_{3}=1$ & $\mu_{3}=2$ & $R_{3}=4$ \\
\hline
\end{tabular}

The original policy is as below. The voice preservation slot is set to be 0, Data preservation slot is set to be 0 , the control method is preemptive (i.e. data users must stop transmission immediately to allow voice users to use the slots if all slots are occupied.). The changed states are shown as below: 
TABLE IV. THE CHANGED STATES OF EXAMPLE

\begin{tabular}{|l|l|l|}
\hline State & Old decision & New decision \\
\hline$(0,0,3)$ & All & voice\&2-slot \\
\hline$(0,1,3)$ & Voice\&single & Voice \\
\hline$(0,5,0)$ & All & Voice\&2-slot \\
\hline$(0,6,0)$ & All & Voice\&2-slot \\
\hline$(0,7,0)$ & Voice\&single & Voice \\
\hline$(1,0,3)$ & Voice\&single & Voice \\
\hline$(1,5,0)$ & All & Voice\&2-slot \\
\hline$(1,6,0)$ & Voice\&single & Voice \\
\hline$(2,5,0)$ & Voice\&single & Voice \\
\hline$(5,1,1)$ & Voice & No \\
\hline$(6,0,1)$ & Voice & No \\
\hline$(7,1,0)$ & Voice & No \\
\hline
\end{tabular}

The results are: 3 iterations to achieve the optimal policy, 12 decisions are changed, Original profit is 79.10878, Maximum profit is 79.6666.

In this example, we assume voice users have higher arrival rate and higher departure rate and the highest revenue. Thus the system will grant voice users as possible to get the optimum long-term revenue. In some states the system will reject data users request because they will stay in system longer and generate less revenue than voice users. The computational result is consistent with our expectation. In some states even there are still some slots for all users but the system only grant voice users due to their long-term high revenue.

TABLE V. OTHER POLICIES AND THEIR CORRESPONDING REVENUES

\begin{tabular}{|l|l|l|l|}
\hline Policy & Revenue & Policy & Revenue \\
\hline 1,7 & 51.22687 & 2,4 & 75.6151 \\
\hline 2,6 & 65.324 & 2,3 & 77.84588 \\
\hline 3,5 & 71.46927 & 2,2 & 79.05113 \\
\hline 4,4 & 74.91467 & 2,1 & 79.43186 \\
\hline 5,3 & 76.88034 & 3,4 & 75.34375 \\
\hline 6,2 & 77.34731 & 3,3 & 77.67289 \\
\hline 7,1 & 77.042 & 3,2 & 78.91668 \\
\hline 1,6 & 65.06944 & 3,1 & 79.32901 \\
\hline 1,5 & 71.79738 & 4,3 & 77.39511 \\
\hline 1,4 & 75.53198 & 4,2 & 78.71202 \\
\hline 1,3 & 77.79269 & 4,1 & 79.17453 \\
\hline 1,2 & 79.0042 & 5,2 & 78.34699 \\
\hline 1,1 & 79.39543 & 5,1 & 78.90591 \\
\hline 2,5 & 71.86972 & 6,1 & 78.18542 \\
\hline
\end{tabular}

In this example only 12 decisions are changed, which means the original policy is good for this situation. So the difference between maximum profit and original profit is not much. We can compare with the other policy to show that our result is the best policy. Table $\mathrm{V}$ lists the other policies and their corresponding revenue. $(1,7)$ means that the system reserves one slot for voice users and reserves 7 slots for data users, including single-slot and two-slot data users.

According to above example, we can find out that if the voice users have higher arrival and departure rate and higher revenue, the original policy is good. The system can grant users as long as there are free slots and the difference between the original profit and maximum profit is small. But when the data users have higher arrival rate and departure rate and higher revenue, the original policy is not suitable. The system should grant users carefully because the maximum profit is much greater than the original one.

\section{CONCLUSION}

In this paper, we developed a mathematical model to describe the LTE time slot allocation problem. After completing the work, there are still other issues to be done for LTE time slot allocation systems. First, in our model we only consider three classes of users. But in real world, other users are also possible. Maybe there will be a user who need three-slot simultaneously to transmit his data. Demands for more slots are also possible. Considering these classes of users will make our model become more complicated, but in order to improve the accuracy of the model, it is necessary.

Second, the total time slots of the system might be greater than 8. In real world we can use some techniques to "combine" the slots. Therefore, the total slots may be 16 or 24 , even greater. However, to improve the accuracy of our model, this consideration is still necessary.

\section{REFERENCES}

[1] S. B.Rejeb, N.Nasser, S.Tabbane, "A novel resource allocation scheme for LTE network in the presence of mobility," Journal of Network and Computer Applications, vol.46, 2014, pp. 352-361.

[2] L. Mogan, V. Mantharachalam, G. Kalaichelvi, et al. "Dynamic Markov Model based spectrum sharing and band aggregation for LTE-700 MHz band in India," Proc. 2015 3rd International Conference on. IEEE Signal Processing, Communication and Networking (ICSCN), 2015, pp. 1-6.

[3] N. A. Amirrudin, S. H.Ariffin, N. Malik, "Mobility prediction via Markov model in LTE femtocell," Internation Journal of Computer Application, vol. 65, 2013, pp. 40-44.

[4] X.Xiang, C.Lin, X.Chen, et al. "Toward optimal admission control and resource allocation for LTE-A femtocell uplink," IEEE Transactions on Vehicular Technology, vol. 64 2015, pp. 3247-3261.

[5] K. B.Ali, M. S.Obaidat, F.Zarai, et al. "Markov model-based adaptive CAC scheme for 3GPP LTE femtocell networks," 2015 IEEE International Conference on Communications (ICC). IEEE, 2015, pp. 6924-6928.

[6] T.Tsang, M.Bilal, P. M. Chan, et al. "Performance Analysis for LTE Networks with Markov Decision Process," Cyber Journals: Multidisciplinary Journals in Science and Technology, Journal of Selected Areas in Telecommunications, August Edition, vol. 3, 2013, pp.

$1-10$. 